\title{
Methods for simulating the readout of lengths and angles in laser interferometers with Gaussian beams
}

\author{
Gudrun Wanner $^{\mathrm{a}, \mathrm{b}, *}$, Gerhard Heinzel ${ }^{\mathrm{a}, \mathrm{b}}$, Evgenia Kochkina ${ }^{\mathrm{a}, \mathrm{b}}$, Christoph Mahrdt ${ }^{\mathrm{a}, \mathrm{b}}$, \\ Benjamin S. Sheard ${ }^{\mathrm{a}, \mathrm{b}}$, Sönke Schuster ${ }^{\mathrm{a}, \mathrm{b}}$, Karsten Danzmann ${ }^{\mathrm{a}, \mathrm{b}}$ \\ ${ }^{a}$ Max-Planck-Institute for Gravitational Physics (Albert-Einstein-Institute), Callinstr. 38, D-30167 Hanover, Germany \\ ${ }^{\mathrm{b}}$ QUEST Centre of Quantum Engineering and Space-Time Research, Leibniz University of Hanover, Hanover, Germany
}

\section{A R T I C L E I N F O}

\section{Article history:}

Received 2 December 2011

Received in revised form

11 July 2012

Accepted 30 July 2012

Available online 21 August 2012

Keywords:

Interferometry

Simulation

Differential wavefront sensing

Phase demodulation

Heterodyne

Homodyne

\begin{abstract}
A B S T R A C T
In this paper methods to simulate the signals in laser interferometers are proposed. The central part deals with the computation of the photocurrent, subsequent phase demodulation and finally the generation of interferometer signals, such as the longitudinal phase readout, differential wavefront sensing signal, differential power sensing and contrast. Here, fundamental Gaussian beams without astigmatism are assumed. The methods are validated in several examples by comparison with experimental data, with analytical results as well as with an intuitively predictable system.
\end{abstract}

(c) 2012 Elsevier B.V. All rights reserved.

\section{Introduction}

The scientific use of laser interferometers for precision length measurements has become extensive in the past decades. For instance in the field of gravitational wave detection, heterodyne and homodyne interferometers are used to sense tiny changes in the optical pathlengths in order to detect and chart gravitational waves [1-3]. Another example is the use of laser interferometers for spaceborne mapping of the Earth's gravity field [4,5]. In such interferometers, a laser beam is reflected from a mirror, which we call test mass following the convention in gravitational wave missions. The beam is then interfered with a reference laser beam (see Fig. 1). Often, the two beams differ in frequency (heterodyne interferometers) and we will assume that for the discussion, although the methods can also be applied to homodyne interferometers. The resulting interference pattern is recorded with a photodiode which senses any phase shift in the resulting sinusoidal beat note. This phase of the signal is then measured using a phasemeter and converted into a length. The resulting signal is the "longitudinal pathlength signal" (LPS) which senses any longitudinal movement of the test mass with high accuracy.

\footnotetext{
* Corresponding author at: Max-Planck-Institute for Gravitational Physics (Albert-Einstein-Institute), Callinstr. 38, D-30167 Hanover, Germany.

E-mail addresses: gudrun.wanner@aei.mpg.de (G. Wanner), gerhard.heinzel@aei.mpg.de (G. Heinzel).
}

A tilt of the test mass typically causes the beam reflected from the test mass, the so-called measurement beam, to shift away from the center of the photodiode. If the detector is a quadrant photodiode (QPD), this beam walk can be sensed by the imbalance of the sensed power of the four quadrants. The resulting signal is the "differential power sensing" (DPS) or ratiometric signal [6]. It can be used to measure tilts of the test mass over a large angular range, limited only by the size of the photodiode or other apertures.

A tilt of the test mass also tilts the phasefront of the measurement beam with respect to the wavefront of the reference beam (see Fig. 1 for an illustration). This causes an imbalance of the phase signals on the various quadrants of the QPD. The difference between these phase signals can be processed to produce the "differential wavefront sensing" (DWS) signals $[7,8]$. This is a high gain readout of the angle between the wavefronts and thereby a high precision readout of test mass angular movements. In interferometers where the DPS signal does not sense test mass rotations because imaging optics are used to suppress resulting beam walk on the photoreceiver, the DWS signal can still be used for angular readout.

Finally, the contrast or fringe visibility of the interference pattern is a typical interferometer property which gives general information about the alignment status of the interferometer.

The prediction of these signals in laser interferometers is cumbersome, since there exists no simple software tool or analytical method to generate all these alignment signals for fundamental Gaussian 

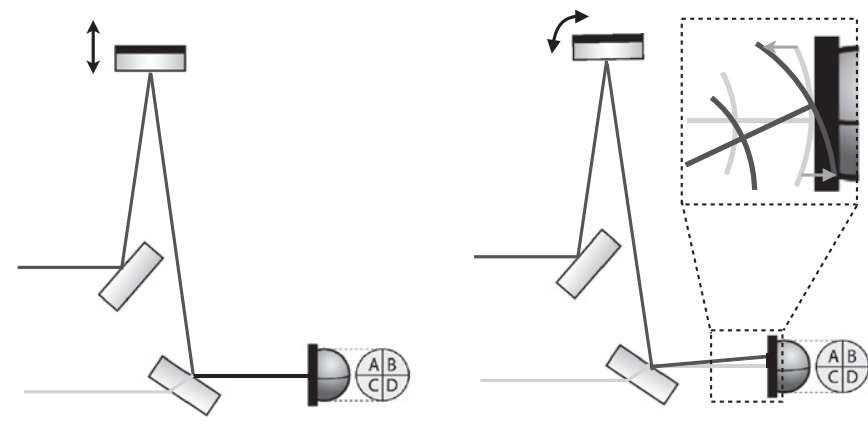

Fig. 1. Typical interferometer with test mass (top mirror) and post-processing of the photocurrent using phasemeter and computer (left graph). The right hand side figure sketches how a test mass tilt causes a DWS signal: the measurement beam wavefront is ahead of the reference wavefront on quadrants $C$ and D but lags behind the reference wavefront on quadrants $A$ and B. This effect causes unequal phase signals on those quadrants and thereby a DWS signal.

beam input. Methods for tracing beams through optical systems are known for decades and also methods for phase demodulation from a photocurrent have been previously discussed in detail (for instance in [9-14]). We present now how all these methods can be combined to predict interferometric readout signals like those introduced above, starting from the point of beam definition, tracing through arbitrary three dimensional optical systems to generation of a virtual photocurrent and the subsequent phase demodulation.

In this paper, we describe the basic methods needed for typical laser interferometers. That means in particular, that the following assumptions are made: Laser beams are circular (i.e. non-astigmatic) fundamental Gaussian beams (i.e. in TEM00 mode). Nonpolarizing interferometers are used and all defined surfaces are either flat or spherical and have no structure or defects. Generalization of the methods to, for instance, higher order beam modes (for example needed if a laser beam is clipped by an aperture) or treatment of astigmatism in three dimensions (including nonnormal incidence at curved surfaces) is work in progress and will be described in separate papers.

Outline. In Section 2 the main methods are presented, which describe the computation of longitudinal and alignment signals. In Section 3 several examples are given, comparing the numerical method from Section 2 with experimental data (Section 3.1) and analytical solutions (Sections 3.2 and 3.3). Since an analytical prediction of the LPS in the general case is currently not possible, one intuitively predictable example is given in Section 3.4.

\section{Interferometer signal prediction}

\subsection{Beam impact on detector and resulting photocurrent}

In this paper, methods for simulating two beam interferometers are described. It is thus assumed, that after the ray tracing part is completed, there are two beams impinging on a detector: a measurement beam $\mathcal{B}_{M}$ and a reference beam $\mathcal{B}_{R}$. These beams interfere and cause a time varying photocurrent $J(t)$ which is proportional to the sensed beam power $P_{S}$ on the detector surface $S[15,16]:$

$J=\frac{\eta e}{\hbar \omega} P_{S}(t)=\rho P_{S}(t)$

$P_{S}(t)=\int \mathrm{d} S I(t)=\frac{1}{2 Z} \int \mathrm{d} S\left|E_{R}(t)+E_{M}(t)\right|^{2}$

where $\rho$ is the photodiode responsivity, $\eta$ the quantum efficiency, $Z$ the impedance of the medium, and $I$ is the local intensity. Neither the photodiode responsivity $\rho$ nor the impedance $Z$ of the medium are needed for the phase demodulation and signal computation. These factors are given only for completeness. It is however possible to set $\rho=2 Z=1$ in a numerical computation.

In order to simulate this photocurrent - or effectively the power sensed by the photodiode - the electric fields $E_{R}$ and $E_{M}$ need to be computed. If $\left(x_{b}, y_{b}, z_{b}\right)$ is a point described in the beamfixed coordinate system, the electric field of a Gaussian beam segment in fundamental mode propagating in $z$-direction can be written as $[17,18]$

$E\left(r_{b}, z_{b}, t\right)=E_{0} \frac{w_{0}}{w\left(z_{b}\right)} \exp \left(\frac{-r_{b}^{2}}{w\left(z_{b}\right)^{2}}\right) \exp \left(\frac{-i k r_{b}^{2}}{2 R\left(z_{b}\right)}+i \eta\left(z_{b}\right)-i k z\right) \exp (i \omega t)$

with

$r_{b}=\sqrt{x_{b}^{2}+y_{b}^{2}}$,

wavenumber $k=2 \pi / \lambda$, angular laser frequency $\omega$, radius of curvature $R\left(z_{b}\right)$, Gouy phase $\eta\left(z_{b}\right)$ and the spot size $w\left(z_{b}\right)$ defined from the Rayleigh range $z_{0}$ and the distance $z_{b}$ of the current position from the beam waist by

$\frac{1}{R\left(z_{b}\right)}=\frac{z_{b}}{z_{b}^{2}+z_{0}^{2}}, \quad \frac{1}{w\left(z_{b}\right)^{2}}=\frac{1}{w_{0}^{2}} \frac{z_{0}^{2}}{z_{b}^{2}+z_{0}^{2}}$.

It is convenient to replace the constant $E_{0}=E\left(r_{b}=0, z_{b}=0\right)$ by the total beam power $P$ :

$P=\frac{1}{2 Z} 2 \pi \int_{0}^{\infty} \mathrm{d} r_{b} r_{b}\left\|E\left(r_{b}, z_{b}\right)\right\|^{2}=\frac{1}{2 Z} \frac{\pi w_{0}^{2} E_{0}}{2}$

$\leftrightarrow E_{0}=\sqrt{2 Z} \sqrt{\frac{2 P}{\pi w_{0}^{2}}}$

The phase contribution ikz in Eq. (3) is given with respect to the local waist position. We replace this phase by $i k s$ where $s$ is the optical pathlength propagated from a suitable reference point. This is necessary in order to compare situations when the waist position moves (for instance by shifting curved optics). This argumentation is equally valid for the Gouy phase, which needs to be accumulated in order to avoid unphysical phase jumps at curved surfaces (cf. Fig. 2). The electric field equation thus reads

$E\left(r_{b}, z_{b}, t\right)=\sqrt{2 Z} \sqrt{\frac{2 P}{\pi w\left(z_{b}\right)^{2}}} \exp \left(\frac{-r_{b}^{2}}{w\left(z_{b}\right)^{2}}\right) \exp \left(\frac{-i k r_{b}^{2}}{2 R\left(z_{b}\right)}+i \eta_{\mathrm{ac}}-i k s\right) \exp (i \omega t)$,

$E\left(r_{b}, z_{b}, t\right)=E\left(r_{b}, z_{b}\right) \exp (i \omega t)$.

For the integration over the detector surface, it is convenient to describe the electric field in the detector coordinate frame. For the
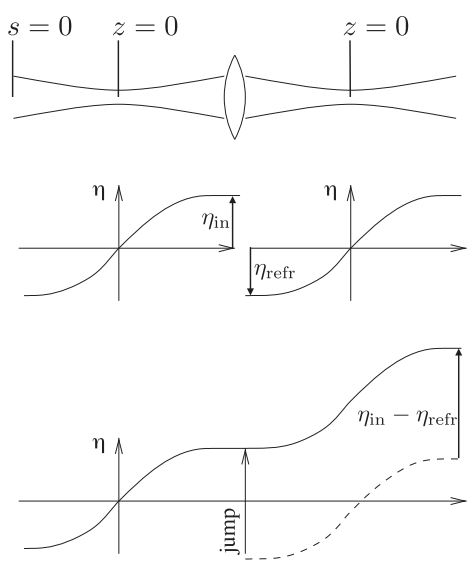

Fig. 2. Accumulation of the Gouy phase during propagation and refraction. 


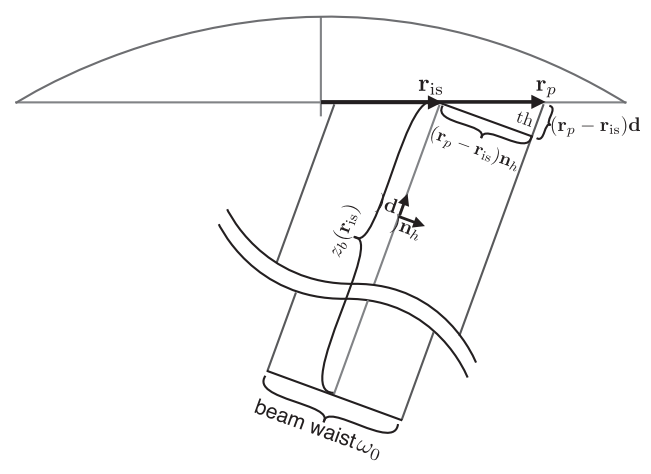

Fig. 3. Coordinate transformation in the electric field, when a beam impinges on a photodetector. Here, $\mathbf{r}_{p}$ is an arbitrary point on the detector surface, $\mathbf{r}_{\text {is }}$ is the intersection point between beam and detector and $\mathbf{d}$ is the direction of propagation of the beam.

corresponding coordinate transformation we need the intersection point $\mathbf{r}_{\text {is }}$ of ray and detector surface and consider the electric field at a point $\mathbf{r}_{\mathrm{p}}$ on the detector. Both $\mathbf{r}_{\mathrm{p}}$ and $\mathbf{r}_{\mathrm{is}}$ are on the detector, such that they are two dimensional with origin at the detector center.

In the beam-fixed coordinate system the direction of propagation d forms an orthonormal basis with two normal vectors, called "horizontal" $\mathbf{n}_{h}$ and "vertical" $\mathbf{n}_{v}$. Since all beams are assumed here to be circular, $\mathbf{n}_{h}$ and $\mathbf{n}_{v}$ are not uniquely defined but can be chosen as convenient. The coordinate transformation for the electric field of Eq. (3) is then given by

$x_{b}\left(\mathbf{r}_{p}\right)=\left(\mathbf{r}_{p}-\mathbf{r}_{\mathrm{is}}\right) \mathbf{n}_{h}$,

$y_{b}\left(\mathbf{r}_{p}\right)=\left(\mathbf{r}_{p}-\mathbf{r}_{\mathrm{is}}\right) \mathbf{n}_{v}$,

$z_{b}\left(\mathbf{r}_{p}\right)=\left(\mathbf{r}_{p}-\mathbf{r}_{\text {is }}\right) \mathbf{d}+z_{b}\left(\mathbf{r}_{\text {is }}\right)$

as illustrated in Fig. 3. The optical pathlength $s$ accumulated in the optical system during the ray tracing procedure is valid for the intersection point $\mathbf{r}_{\text {is }}$ and the intersection plane spanned by $\mathbf{n}_{v}$ and $\mathbf{n}_{h}$. In case of non-normal incidence the additional pathlength $\left(\mathbf{r}_{p}-\mathbf{r}_{\text {is }}\right) \mathbf{d}$ for all points $\mathbf{r}_{p}$ on the detector surface needs to be accounted for by substituting:

$s\left(\mathbf{r}_{p}\right)=s\left(\mathbf{r}_{\text {is }}\right)+\left(\mathbf{r}_{p}-\mathbf{r}_{\text {is }}\right) \mathbf{d}$.

The electric field is thus fully defined in beam-fixed coordinates for each beam and for any point $\mathbf{r}_{p}$ on the detector surface. Therefore, if two beams impinge on a detector, the beam power sensed by the detector can be computed by a simple integral over the detector surface (cf. Eq. (2)). In general, the integrals can only be evaluated numerically. In that case an adaptive integration package like DCUHRE [19] can be used. Since DCUHRE is designed for integrations over rectangular grids, circular photodiodes can be implemented either by nested one dimensional integrals or by specifying a circular filter function $F$, which is multiplied to the intensity $I$ in Eq. (2):

$I_{\text {circular }}=I F, \quad F=1-\Theta\left(r_{b}-r_{c}\right)$,

where $\Theta\left(r_{b}\right)$ is the Heaviside step function, and $r_{c}$ is the radius of the photoactive area. It can be useful to use a differentiable approximation of the Heaviside step function. One option for such an approximation is

$F=0.5\left[1-\operatorname{erf}\left(\frac{r_{c}-r_{b}}{W}\right)\right]$.

Here, $W$ is a constant which can be adapted to the current problem in order to reduce the numerical integration time. For instance $W=0.001 r_{c}$ can be chosen.

After the integration (Eq. (2)) is performed, the power sensed by the detector or equivalently the resulting photocurrent is known.

\subsection{Homodyne and heterodyne interferometry}

Eq. (3) can be rewritten using a phase $\Phi$ and an amplitude $A$ :

$E\left(r_{b}, z_{b}, t\right)=A\left(r_{b}, z_{b}\right) \exp \left(i \omega t-i \Phi\left(r_{b}, z_{b}\right)\right)$

with

$A\left(r_{b}, z_{b}\right)=\sqrt{2 Z} \sqrt{\frac{2 P}{\pi w\left(z_{b}\right)^{2}}} \exp \left(\frac{-r_{b}^{2}}{w\left(z_{b}\right)^{2}}\right)$,

$\Phi\left(r_{b}, z_{b}\right)=\frac{k r_{b}^{2}}{2 R\left(z_{b}\right)}-\eta\left(z_{b}\right)+k s$.

For clarity, the dependence of the beam coordinates $r_{b}$ and $z_{b}$ will be omitted in the subsequent equations. The local laser intensity resulting from the interference of two such laser beams is then

$$
\begin{aligned}
I & =\frac{1}{2 Z}\left|A_{R} \exp \left(i \omega_{R} t-i \Phi_{R}\right)+A_{M} \exp \left(i \omega_{M} t-i \Phi_{M}\right)\right|^{2} \\
& =\frac{1}{2 Z}\left(A_{R}^{2}+A_{M}^{2}\right)\left[1+\frac{2 A_{M} A_{R}}{A_{M}^{2}+A_{R}^{2}} \cos (\Delta \omega t-\Delta \Phi)\right] \\
& =\bar{I}\left[1+c_{I} \cos (\Delta \omega t-\Delta \Phi)\right],
\end{aligned}
$$

where $\bar{I}$ is the time averaged local intensity and $c_{I}$ the local contrast (fringe visibility). The phase difference $\Delta \Phi$ contains the pathlength difference between the two beams. The power sensed by the active surface $S$ of a detector is then given by

$P_{S}=\int \mathrm{d} S I$,

which has the general form

$P_{S}=\bar{P}[1+c \cos (\Delta \omega t-\phi)]$.

This follows from the addition theorems of trigonometric functions or the fact that the superposition of general sinusoids with equal frequency is again a sinusoid of the same frequency: $\sum_{n} A_{n} \sin \left(\omega t+\phi_{n}\right)=A \sin (\omega t+\phi)$.

\subsection{Phase demodulation}

With the procedures of the previous section, a virtual photocurrent can be computed. It corresponds to one certain but yet unknown combination of $\bar{P}, c, \phi$ in Eq. (22). Our next aim is to determine $\bar{P}, c, \phi$ from a given sensed power $P_{S}$ or equivalently a given photocurrent $J$. This virtual photocurrent can be processed with the same methods as a real photocurrent in an experiment. We describe here the methods of phase demodulation performed with the phasemeters in the LISA Pathfinder experiment [6]. These methods are based upon a few simple integrals over the sensed power $P_{S}$ in a heterodyne interferometer $(\Delta \omega \neq 0)$. The first integral is a plain averaging of the power over one period, which results in the mean power $\bar{P}$ :

$\frac{1}{2 \pi} \int_{0}^{2 \pi} \mathrm{d}(\Delta \omega t) P_{S}=\underbrace{\bar{P}}_{=1} \underbrace{\frac{1}{2 \pi} \int_{0}^{2 \pi} \mathrm{d}(\Delta \omega t)[1+c \cos (\Delta \omega t-\phi)]}=\bar{P}$.

Additionally, the following two integrals are needed:

$C^{Q}:=\frac{1}{\pi} \int_{0}^{2 \pi} \mathrm{d}(\Delta \omega t) P_{S} \cos (\Delta \omega t)$

$C^{I}:=\frac{1}{\pi} \int_{0}^{2 \pi} \mathrm{d}(\Delta \omega t) P_{S} \sin (\Delta \omega t)$.

If $P_{S}$ has the form given in Eq. (23), $C^{l}$ and $C^{Q}$ evaluate to

$C^{Q}=\bar{P} c \cos (\phi)$, 
$C^{I}=\bar{P} C \sin (\phi)$.

The values $C^{I}$ and $C^{Q}$ define the complex amplitude $C$ :

$C:=C^{Q}+i C^{I}$,

which has according to Eqs. (27) and (28) the following value:

$C=\bar{P} c \exp (i \phi)$.

The contrast $c$ and phase $\phi$ are thus extracted from the integrals (25) and (26) using

$c=\frac{\sqrt{\left(C^{Q}\right)^{2}+\left(C^{I}\right)^{2}}}{\bar{P}}=\frac{|C|}{\bar{P}}$,

$\phi=\arctan \left(\frac{C^{I}}{C^{Q}}\right)=\arg (C)$.

This method uses integration over continuous data. In order to simulate the experimental sampling procedure, the given equations need to be discretized. This can be performed using the $\mathrm{N}$-bucket algorithm (for instance [9-14]). Here, instead of a frequency shift $\Delta \omega$ a phase shift $2 \pi n / N$ is introduced in one of the two impinging beams, and Eq. (22) is evaluated $N$ times:

$P_{S}^{n}=\frac{1}{2 Z} \int \mathrm{d} S\left\|E_{R}\left(r_{b}, z_{b}\right)+E_{M}\left(r_{b}, z_{b}\right) \exp \left(i \frac{2 \pi n}{N}\right)\right\|^{2}, \quad n=0, \ldots, N-1$,

where any number of $N \geq 3$ [14] can be chosen. Since the virtual photocurrent is typically free of noise, there is no need to choose a large $N$ and we usually use $N=5$ to allow consistency checks. The $\mathrm{N}$-bucket algorithm can be used in heterodyne as well as in homodyne interferometers. For heterodyne interferometers, the time dependent photocurrent would be sampled at specific times:

$t(n)=\frac{2 \pi n}{N \omega_{\text {het }}}$.

However, in homodyne interferometers where $\Delta \omega$ is zero, the additional phase shift of $2 \pi n / N$ has to be introduced explicitly in the experiment.

The mean power on the surface $S$ is now computed by

$\bar{P}_{S}=\frac{1}{N} \sum_{n=0}^{N-1} P_{S}^{n}$

The equivalents to Eqs. (25) and (26) are

$C_{S}^{Q}:=\frac{2}{N} \sum_{n=0}^{N-1} P_{S}^{n} \cos \left(\frac{2 \pi n}{N}\right)=\bar{P}_{S} c_{S} \cos \left(\phi_{S}\right)$,

$C_{S}^{I}:=\frac{2}{N} \sum_{n=0}^{N-1} P_{S}^{n} \sin \left(\frac{2 \pi n}{N}\right)=\bar{P}_{S} c_{S} \sin \left(\phi_{S}\right)$

and Eqs. (29)-(31), that is the contrast $c_{S}$ and phase $\phi_{S}$ sensed by a detector with surface $S$, now becomes

$C_{S}:=C_{S}^{Q}+i C_{S}^{I}=\bar{P}_{S} c_{S} \exp \left(i \phi_{S}\right)$

$c_{S}=\frac{\sqrt{\left(C_{S}^{Q}\right)^{2}+\left(C_{S}^{I}\right)^{2}}}{\bar{P}_{S}}=\frac{\left|C_{S}\right|}{\bar{P}_{S}}$,

$\phi_{S}=\arctan \left(\frac{C_{S}^{I}}{C_{S}^{Q}}\right)=\arg \left(C_{S}\right)$.

The consistency and accuracy can be checked by testing whether

$P_{S}^{n}=\bar{P}_{S}\left[1+c_{S} \cos \left(\frac{2 \pi n}{N}-\phi_{S}\right)\right]$.
From the results (mean power $\bar{P}_{S}$, contrast $c_{S}$, and phase signal $\phi_{S}$ ) the most important one usually is the phase signal which can be converted to a length by

LPS $=\phi_{S} / k$

defining the longitudinal pathlength signal "LPS". This signal is the best estimate available in the experiment for the optical pathlength difference between the two interferometer arms $\left(s_{M}-s_{R}\right)$ but it is also influenced by the wavefront curvature and Gouy phase (Eq. (18)) and detector shape (Eq. (33)).

An alternative way to find the mean power $\bar{P}$, contrast $c$ and phase $\phi$ is computing the subsequent three integrals:

$\bar{P}=\frac{1}{2 Z} \int \mathrm{d} S\left(\left\|E_{M}\left(r_{b}, z_{b}\right)\right\|^{2}+\mid E_{R}\left(r_{b}, z_{b}\right) \|^{2}\right)$,

$C^{Q}=\frac{1}{2 Z} \int \mathrm{d} S 2 \mathfrak{R}\left[E_{M}\left(r_{b}, z_{b}\right) E_{R}^{*}\left(r_{b}, z_{b}\right)\right]$,

$C^{I}=\frac{1}{2 Z} \int \mathrm{d} S 2 \mathfrak{J}\left[E_{M}\left(r_{b}, z_{b}\right) E_{R}^{*}\left(r_{b}, z_{b}\right)\right]$

and using Eqs. (31) and (32) to compute the contrast $c$ and phase $\phi$. This method requires comparable numerical effort but lacks the possibility of consistency checking.

\subsection{Separation of macroscopic and microscopic phase}

The pathlength $s\left(\mathbf{r}_{\text {is }}\right)$ accumulated during the beam propagation through the setup (Eq. (13)) is typically in the range of millimeters to meters. We therefore call $k s\left(\mathbf{r}_{\text {is }}\right)$ the macroscopic phase. All remaining contributors to the phase $\Phi$ in Eq. (18) are usually in the order of picometer to micrometer (depending on the setup) and are therefore referred to as microscopic phase. In order to avoid numerical precision problems, it is necessary to separate the macroscopic and microscopic phases. This can be performed by omitting $s\left(\mathbf{r}_{\text {is }}\right)$ from the electrical field in Eq. (8), such that only the additional pathlength originating from a tilt of the beam relative to the detector surface is accounted for

$s\left(\mathbf{r}_{p}\right)=\left(\mathbf{r}_{p}-\mathbf{r}_{\text {is }}\right) \cdot \mathbf{d}$.

This is compensated by adding $s\left(\mathbf{r}_{\text {is }}\right)$ to the computed longitudinal pathlength signal by

$\mathrm{LPS}=s\left(\mathbf{r}_{\mathrm{is}}\right)+\phi_{S} / k$.

This method is valid because the macroscopic phase $k s$ is constant over the detector surface $S$, due to the coordinate transformation performed in Eq. (13).

\subsection{Alignment signals for quadrant photodiodes}

For a quadrant photodiode (QPD), the surface $S$ corresponds to any of the four quadrants. Therefore, four sets of signals (mean power $\bar{P}$, contrast $c$ and phase signal $\phi$ ) are generally available for quadrant detectors. By combining these signals, a larger variety of signals can be defined. First, the power sensed by the various quadrants can be compared, which results in a measure of the beam centroid position. The resulting signals are called horizontal and vertical differential power sensing signal (DPS signal) throughout this paper:

$\mathrm{DPS}_{h}:=\frac{\bar{P}_{l}-\bar{P}_{r}}{\bar{P}_{l}+\bar{P}_{r}}=\frac{\bar{P}_{A}+\bar{P}_{C}-\bar{P}_{B}-\bar{P}_{D}}{\bar{P}_{A}+\bar{P}_{B}+\bar{P}_{C}+\bar{P}_{D}}$,

$\mathrm{DPS}_{v}:=\frac{\bar{P}_{t}-\bar{P}_{b}}{\bar{P}_{t}+\bar{P}_{b}}=\frac{\bar{P}_{A}+\bar{P}_{B}-\bar{P}_{C}-\bar{P}_{D}}{\bar{P}_{A}+\bar{P}_{B}+\bar{P}_{C}+\bar{P}_{D}}$,

where the indices $\{l, r, t, b\}$ label the \{left, right, top and bottom $\}$ halves of the quadrant diode, while $\{A, B, C, D\}$ label the quadrants according to Fig. 4 . These signals are a measure of the beam centroid position on the QPD. 
Similar to the definition of the phase signal and the LPS on a single element detector, it is possible to define a longitudinal pathlength signal for a quadrant detector, by combining the four segments. One possibility is to use the argument of the sum of all complex amplitudes:

$$
\begin{aligned}
& \mathrm{LPS}^{\mathrm{LPF}}=\frac{1}{k} \phi^{\mathrm{LPF}}:=s+\frac{1}{k} \arg \left(C_{A}+C_{B}+C_{C}+C_{D}\right), \\
& \mathrm{LPS}^{\mathrm{LPF}}=s+\frac{1}{k} \arctan \\
& \qquad\left(\frac{c_{A} \bar{P}_{A} \sin \phi_{A}+c_{B} \bar{P}_{B} \sin \phi_{B}+c_{C} \bar{P}_{C} \sin \phi_{C}+c_{D} \bar{P}_{D} \sin \phi_{D}}{c_{A} \bar{P}_{A} \cos \phi_{A}+c_{B} \bar{P}_{B} \cos \phi_{B}+c_{C} \bar{P}_{C} \cos \phi_{C}+c_{D} \bar{P}_{D} \cos \phi_{D}}\right) .
\end{aligned}
$$

This procedure to generate an LPS is used for instance in the LISA Pathfinder (LPF) mission [6], such that we call it LPS ${ }^{\text {LPF }}$. However, this is not the only possibility to define an LPS for a QPD. One could, for example, also directly average the phases of the various quadrants: $\left(\phi_{A}+\phi_{B}+\phi_{C}+\phi_{D}\right) / 4$, or apply various weighting factors in the averaging. A comparison of the possible definitions is work in progress and will be discussed in a separate paper.

The phase signals of the various quadrants can be compared in a similar way as the mean power for the DPS signal. The resulting signals are the differential wavefront sensing (DWS) signals [7,8]:

$$
\begin{aligned}
& \mathrm{DWS}_{h}=\phi_{l}-\phi_{r}, \\
& \mathrm{DWS}_{v}=\phi_{t}-\phi_{b} .
\end{aligned}
$$

These signals are a readout of the wavefront tilt between the two interfering beams and therefore are also a high gain readout of test mass tilts or more generally component rotation in the interferometer. An illustration of the corresponding effect is shown in Fig. 1. A comparison between the DPS and DWS signals is listed in Table 1.

Like for the sum phase, the mean phase on the detector halves are generated via the sum of complex amplitudes [6,20]:

$$
\mathrm{DWS}_{h}^{\mathrm{LPF}}:=\arg \left(C_{A}+C_{C}\right)-\arg \left(C_{B}+C_{D}\right)=\arg \left(\frac{C_{A}+C_{C}}{C_{B}+C_{D}}\right),
$$

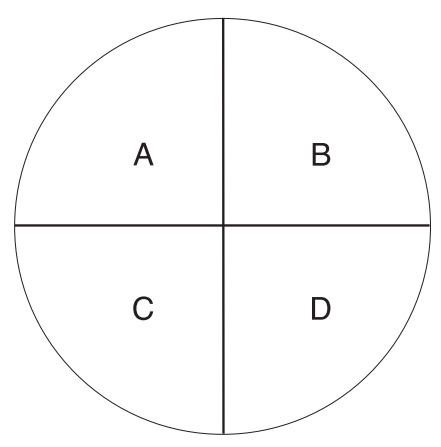

Fig. 4. Labeling of the quadrants of a quadrant photodiode (QPD).
$\mathrm{DWS}_{v}^{\mathrm{LPF}}:=\arg \left(C_{A}+C_{B}\right)-\arg \left(C_{C}+C_{D}\right)=\arg \left(\frac{C_{A}+C_{B}}{C_{C}+C_{D}}\right)$.

The last term is preferable, because it avoids the discontinuity of the $\arg ()$ function on the negative real axis. The macroscopic phase does not contribute to the DWS signals, since it is constant over the entire detector surface-and thus equal on the left and right halves of the diode. Finally, the (mean) contrast for a QPD is defined here as

$c=\frac{\left|C_{A}\right|+\left|C_{B}\right|+\left|C_{C}\right|+\left|C_{D}\right|}{\bar{P}_{A}+\bar{P}_{B}+\bar{P}_{C}+\bar{P}_{D}}$,

which is again an approximation of the sensed contrast of an SEPD which has the same diameter as the QPD.

\subsection{Summary and ray tracing}

In the previous sections, methods to compute interferometer alignment signals have been described. In order to use these equations, two Gaussian beams need to be traced to the detector surface and the intersection point $\mathbf{r}_{\text {is }}$ between each beam and the detector needs to be computed. The complete list of parameters and the corresponding output is sketched in Fig. 5. In case of a two dimensional interferometer, i.e. all rays are lying in one plane, these parameters can be obtained for instance from OptoCad [21] which uses the full equations for reflection and refraction of elliptical Gaussian beams according to [22]. For full three dimensional interferometers, the authors are not aware of a suitable tool to compute these parameters. The development of such a tool is work in progress and currently exists for circular and simple astigmatic beams. The corresponding methods are implemented in $\mathrm{C}$ and $\mathrm{C}++$ libraries which are available on request from the authors.

\section{Examples}

\subsection{Comparison to experimental results}

In the space mission LISA Pathfinder a heterodyne interferometer will be used to sense displacement changes between two free floating test masses which are about $35 \mathrm{~cm}$ apart [1].

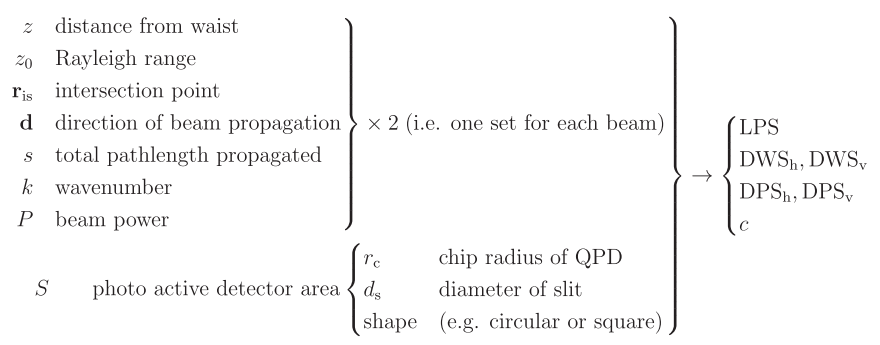

Fig. 5. Parameter input and signal output (LPS, DWS, DPS, $c$ ) corresponding to the procedures described in Section 2 of this paper.

Table 1

Comparison of the properties of DPS and DWS signals.

\begin{tabular}{lll}
\hline Property & DPS & DWS \\
\hline Compares & Power signals & Phase signals \\
Senses & Beam centroid shift on QPD & Relative wavefront tilt \\
Reference & QPD center & Second beam \\
Number of beams needed & One ore more & At least two \\
Tilt readout quality & Low gain, low precision & High gain, high precision \\
Tilt readout w. imaging optics & DPS $0 \rightarrow$ no readout possible & Small (limited by loss of contrast) \\
Range & Wide (limited by QPD size or other apertures) & \\
\hline
\end{tabular}


Additionally, another heterodyne interferometer senses the relative displacement of one of the two test masses with respect to the optical bench. Their signals are labeled "12" and " 1 " respectively. The DWS signals of these interferometers will be used in feedback loops to stabilize the angular jitter of the two test masses. Since the DWS signals are linear in the measurement range, they are typically characterized by coupling factors:

$K_{\varphi_{i}}^{\mathrm{DWS}_{j}}=\frac{\partial \mathrm{DWS}}{\partial \varphi_{i}}$,

where $j$ labels the interferometer (here either "1" or "12") and $i$ the test mass (either " 1 " or "2"). These coupling factors were computed with the numerical procedures described in this paper. In parallel, these coupling factors were measured at the University of Glasgow, using the flight model of the LISA Pathfinder optical bench. The resulting values are listed in Table 2. These values show agreement to a high degree, even though the simulation was performed for the nominal optical bench, i.e. without implementation of tolerances during the assembly of the optical bench. In particular, nominal beam parameters were used for the simulation, and deviations of these beam parameters have a strong impact on the resulting coupling factors.

Additional information regarding the settings of the simulation, the LISA Pathfinder interferometry and the stated values are given in [23].

\subsection{Comparison: numerical and analytical DWS signals}

In some cases it is possible to compute the DWS response of a system analytically, provided that the beam is small compared to the photoactive surface of the QPD, the slit between the quadrants is negligible, and both the relative angle $\alpha$ between the incident beams on a QPD and the static beam offset $x_{0 \text { s }}$ are small. It is thus possible to compare the DWS signal computed with the procedures described in this paper with an analytically computed DWS signal. A simple system fulfilling the listed requirements is given by two interfering beams that propagate freely to a quadrant detector as sketched in Fig. 6. The specific parameters are listed in Table 3.

The DWS signal to first order is according to [24] given by

$\mathrm{DWS}_{\alpha}=\sqrt{\frac{2}{\pi}}\left(k w_{\mathrm{eff}} \alpha\left(1-\frac{z_{\mathrm{tm}}}{R_{M}}\right)-k \frac{w_{\mathrm{eff}} x_{0 \mathrm{~s}}}{R_{M}}\right) F(\sigma)+\mathcal{O}\left(\alpha^{2}, x_{0 \mathrm{~s}}^{2}\right)$.

Table 2

Comparison of measurement values obtained from the University of Glasgow and computed DWS coupling factors for the LISA Pathfinder flight model of the optical bench. Details regarding these values can be found in [23].

\begin{tabular}{lll}
\hline Coupling factor & Numerical simulation & Measurement \\
\hline$K_{\varphi_{1}}^{\mathrm{DWS}_{1}}$ & 4901 & 4985 \\
$K_{\varphi_{1}}^{\mathrm{DWS}_{12}}$ & 4355 & 4529 \\
$K_{\varphi_{2}}^{\mathrm{DWS}_{12}}$ & 5619 & 5155 \\
\hline
\end{tabular}

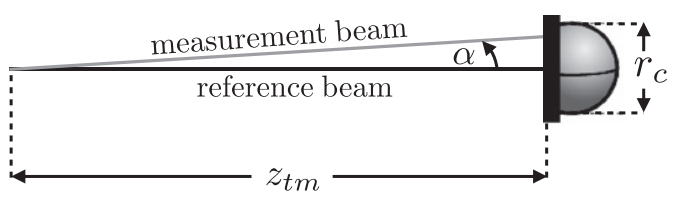

Fig. 6. Sketch of the setup used for Section 3.2.
Table 3

Settings for the comparison between numerical and analytical DWS signal described in Section 3.2. The corresponding results are shown in Fig. 7.

\begin{tabular}{ll}
\hline Parameter & Value \\
\hline Wavelength $\lambda$ & $1064 \mathrm{~nm}$ \\
Beam displacement $x_{0 s}$ at zero beam angle & 0 \\
Waist radius $w_{M}$ of measurement beam & $0.2 \mathrm{~mm}$ or $0.5 \mathrm{~mm}$ \\
Waist radius $w_{R}$ of reference beam & $0.2 \mathrm{~mm}$ \\
$\begin{array}{l}\text { Distance } z_{t m} \text { between pivot } \\
\text { (point of beam definition) and QPD }\end{array}$ & $20 \mathrm{~mm}$ \\
Radius $r_{c}$ of the photoactive surface of the QPD & $5 \mathrm{~mm}$ \\
$\begin{array}{l}\text { Diameter } d_{s} \text { of the slit separating } \\
\text { the quadrants of the QPD }\end{array}$ & $0 \mu \mathrm{m}$ \\
QPD shape & Circular \\
\hline
\end{tabular}

Here, $w_{\text {eff }}$ is the effective beam radius on the photodiode

$\frac{2}{w_{\mathrm{eff}}^{2}}=\frac{1}{w_{M}^{2}}+\frac{1}{w_{R}^{2}}$,

which depends on the spot sizes of the measurement beam $w_{\mathrm{M}}$ and reference beam $w_{\mathrm{R}}$. The variables $F$ and $\sigma$ depend on the relative wavefront curvature and the effective waist:

$F(\sigma)=\frac{1}{\sqrt{2}} \sqrt{\frac{1+\sqrt{1+\sigma^{2}}}{1+\sigma^{2}}}, \quad \sigma=\frac{k w_{\mathrm{eff}}^{2}}{4 R_{\mathrm{rel}}}$.

The relative wavefront curvature $R_{\text {rel }}$ is defined as

$\frac{1}{R_{\text {rel }}}=\frac{1}{R_{R}}-\frac{1}{R_{M}}$,

where $R_{\mathrm{M}}$ and $R_{\mathrm{R}}$ are radii or curvature of the measurement and reference beam at the photodiode surface. Finally, the static lateral beam displacement $x_{0 \text { s }}$ describes the beam displacement for $\alpha=0$ and the distance $z_{\text {tm }}$ specifies the distance between the adjustable mirror and the interference plane.

Result. The resulting DWS signals, computed both numerically and analytically are shown in Fig. 7. For small beam angles, the numerical and analytical procedures generate identical results. This is regarded as a validation of the procedures described in this paper. For large beam angles, the numerical data deviate from the analytical result. This is understandable, since the analytical equation (Eq. (50)) is linearized, in contrast to the numerical procedure. The numerical results are therefore assumed to be more accurate than the analytical result.

\subsection{Comparison: numerical and analytical DPS signals}

The DPS signal as defined in this paper can be computed analytically for a wide set of systems. This results from the definition of the DPS signals via the time averaged power on the various quadrants without any effects of the interference. Therefore, the DPS signal is computed analytically via

$\bar{P}_{S}=\int \mathrm{d} S \bar{I}=\int \mathrm{d} S\left(A_{M}^{2}+A_{R}^{2}\right)$,

where $A_{R}$ and $A_{M}$ are the amplitudes given in Eq. (17) of the measurement and reference beam. It is then assumed that the reference beam is centered on the QPD while the measurement beam moves. For instance, the measurement beam is shifted by $x_{0}$ in the horizontal $x$-direction and has an incident angle of $\alpha$. The coordinate transformation of Eqs. (10) and (11) inserted in Eq. (4) is then

$r_{M}^{2} \rightarrow\left(x-x_{0}\right)^{2} \cos ^{2}(\alpha)+y^{2}$,

$r_{R}^{2} \rightarrow x^{2}+y^{2}$ 

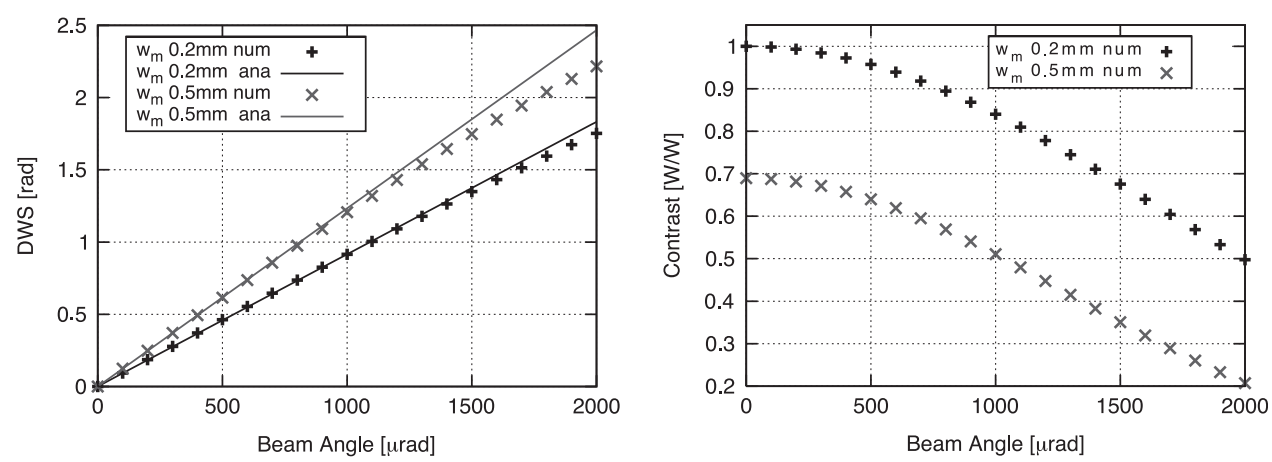

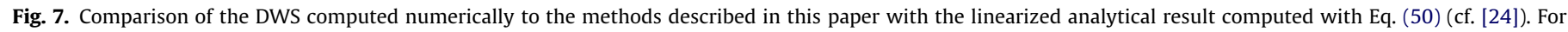
small angles, the numerical and analytical results agree perfectly. For large angles and low contrast the linearized analytical method is not valid.
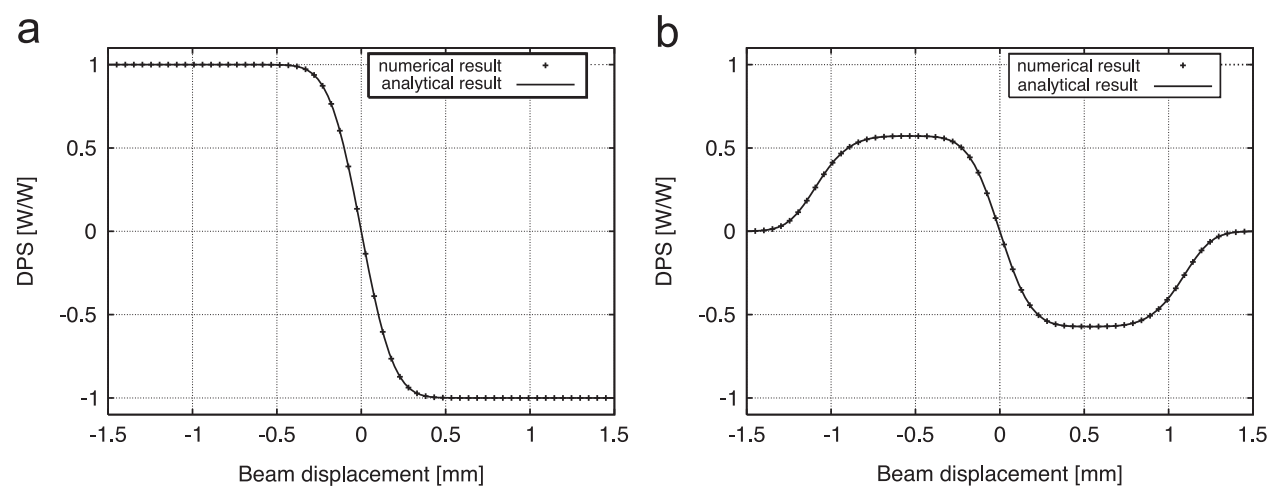

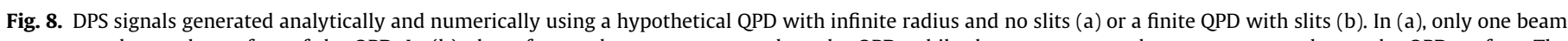

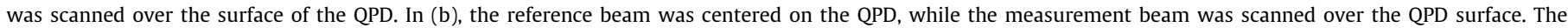
settings used to generate these results are listed in Table 4. For the analytical curves, Eqs. (57) and (58) were used, the incident angle $\alpha$ was set to zero.

The most simple system is a single beam incident on an infinitely large QPD without slits. In this case, the DPS signal is given by a simple error function:

DPS $^{\text {one_beam }}\left(r_{c} \rightarrow \infty, d_{S} \rightarrow 0\right)=-\operatorname{erf}\left(\frac{x_{0} \sqrt{2}|\cos (\alpha)|}{w}\right)$

Fig. 8(a) shows a comparison between this analytical DPS result and the corresponding numerical result, generated with the procedures described in this paper. This figure shows an agreement to a very high degree, even though the curves were generated either numerically using the procedures described in Section 2 thereby including the mixed term $2 \mathfrak{R}\left(E_{M} E_{R}^{*}\right)$ or analytically neglecting this mixed term. The settings used for this simple example are listed in the second column of Table 4. This comparison can also be performed for a more complicated case, with two incident beams and a finite QPD with slits. In this case, the analytical expression for the DPS signal is then given by Eq. (58), where the incident angle $\alpha$ is set to zero to allow clearer arrangement.
Table 4

Settings for the results plotted in Fig. 8. In the system used in Fig. 8(a), only one beam was used and a reference beam was thus not defined (n.d.). In this example, the QPD size was set to infinity to generate the analytical curve (cf. Eq. (57)), for the numerical result, a finite QPD with $10 \mathrm{~mm}$ radius was used. However, this is sufficiently large to generate a perfect agreement in the given range, as shown in Eq. (57).

\begin{tabular}{lll}
\hline Parameter & $\begin{array}{l}\text { Settings for } \\
\text { Fig. 8(a) }\end{array}$ & $\begin{array}{l}\text { Settings for } \\
\text { Fig. 8(b) }\end{array}$ \\
\hline Wavelength $\lambda$ & $1064 \mathrm{~nm}$ & $1064 \mathrm{~nm}$ \\
Measurement beam waist radius & $0.3 \mathrm{~mm}$ & $0.3 \mathrm{~mm}$ \\
Reference beam waist radius & n.d. & $0.3 \mathrm{~mm}$ \\
Location of the pivot & $z=0 \mathrm{~mm}$ & $z=0 \mathrm{~mm}$ \\
Location of measurement beam waist & $z_{M}=0.5 \mathrm{~m}$ & $z_{M}=0.5 \mathrm{~m}$ \\
Location of reference beam waist & n.d. & $z_{R}=0.5 \mathrm{~m}$ \\
Location of the QPD & $z=0.5 \mathrm{~m}$ & $z=0.5 \mathrm{~m}$ \\
Radius $r_{c}$ of the QPD photoactive surface & $10 \mathrm{~mm}$ & $1 \mathrm{~mm}$ \\
QPD slit diameter $d_{S}$ & $0 \mu \mathrm{m}$ & $20 \mu \mathrm{m}$ \\
QPD shape & Square & Square \\
\hline
\end{tabular}

A comparison for such a more typical system is shown in Fig. 8(b), the settings are listed in Table 4 . The analytical and numerical methods generate again perfect agreement.

$$
-\frac{\left(\operatorname{erf}\left[\frac{d_{S}}{\sqrt{2} \mathrm{wz} 1}\right]-\operatorname{erf}\left[\frac{\sqrt{2} r_{c}}{\mathrm{wz} 1}\right]\right)\left(\operatorname{erf}\left[\frac{d_{S}-2 x_{0}}{\sqrt{2} \mathrm{wz} 1}\right]-\operatorname{erf}\left[\frac{d_{S}+2 x_{0}}{\sqrt{2} \mathrm{wz} 1}\right]+\operatorname{erf}\left[\frac{\sqrt{2}\left(x_{0}-r_{c}\right)}{\mathrm{wz} 1}\right]+\operatorname{erf}\left[\frac{\sqrt{2}\left(x_{0}+r_{c}\right)}{\mathrm{wz} 1}\right]\right)}{\left(\operatorname{erf}\left[\frac{d_{S}}{\sqrt{2} \mathrm{wz} 1}\right]-\operatorname{erf}\left[\frac{\sqrt{2} r_{c}}{\mathrm{wz} 1}\right]\right)\left(\operatorname{erf}\left[\frac{d_{S}-2 x_{0}}{\sqrt{2} \mathrm{wz} 1}\right]+\operatorname{erf}\left[\frac{d_{S}+2 x_{0}}{\sqrt{2} \mathrm{wz} 1}\right]+\operatorname{erf}\left[\frac{\sqrt{2}\left(x_{0}-r_{c}\right)}{\mathrm{wz} 1}\right]-\operatorname{erf}\left[\frac{\sqrt{2}\left(x_{0}+r_{c}\right)}{\mathrm{wz} 1}\right]\right)+2\left(\operatorname{erf}\left[\frac{d_{S}}{\sqrt{2} \mathrm{wz2}}\right]-\operatorname{erf}\left[\frac{\sqrt{2} r_{c}}{\mathrm{wz2}}\right]\right)^{2}}
$$




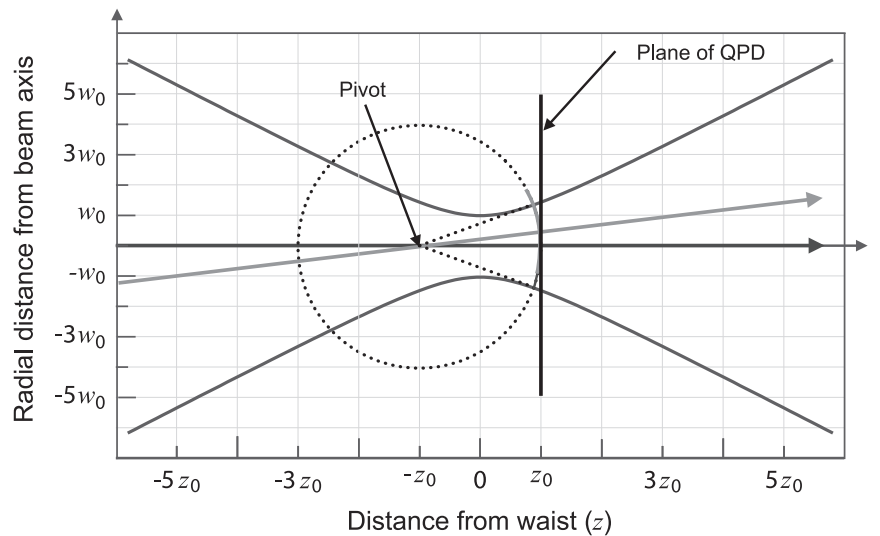

Fig. 9. Gaussian beams and their wavefronts in a system with zero LPS and DWS response. The dotted circle indicates the sphere which represents locally at $z=z_{0}$ the wavefront of both the nominal (gray, on axis) and the tilted (light gray, off axis) beam. A QPD at this location does not sense an LPS or DWS response to the shown beam tilt.

Table 5

Settings for the comparison between numerical and analytical DWS signal described in Section 3.2. The corresponding results are shown in Fig. 7.

\begin{tabular}{ll}
\hline Parameter & $\begin{array}{l}\text { Values for Section } \\
3.4\end{array}$ \\
\hline $\begin{array}{l}\text { Wavelength } \lambda \\
\text { Beam displacement } x_{0 s} \text { at zero beam angle }\end{array}$ & $1064 \mathrm{~nm}$ \\
Rayleigh range $z_{0}$ of both beams & 0 \\
Location of the pivot & $250 \mathrm{~mm}$ \\
Location of both waists (for zero beam angle) & $z=-z_{0}=-250 \mathrm{~mm}$ \\
Location of the QPD & $z=0 \mathrm{~mm}$ \\
Radius $r_{c}$ of the photoactive surface of the QPD & $1 \mathrm{~mm}$ \\
Diameter $d_{S}$ of the slit separating the quadrants of the & $20 \mu \mathrm{mm}$ \\
QPD & \\
QPD shape & Square
\end{tabular}
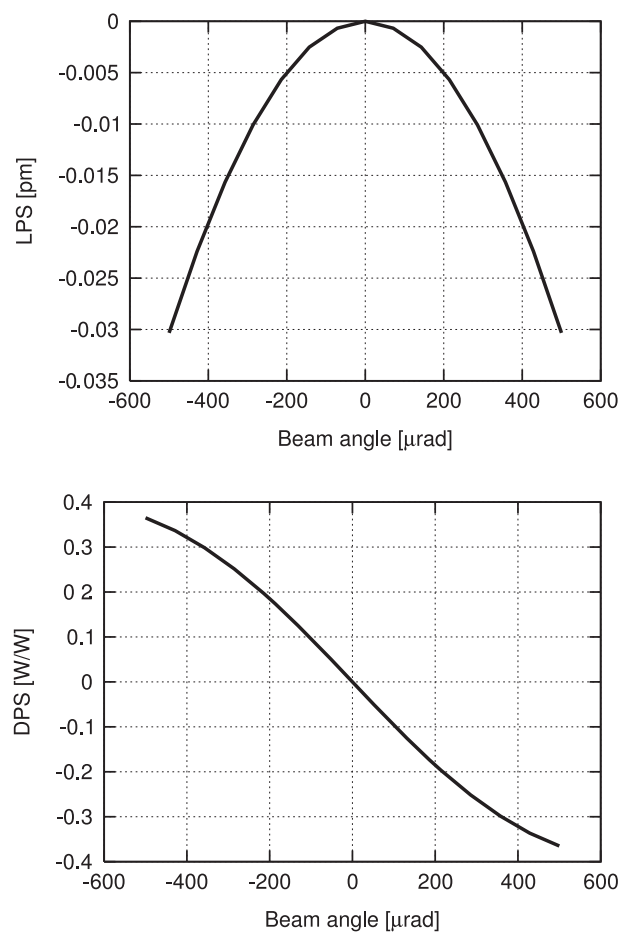

Fig. 10. Signals for the system described in Section 3.4 .
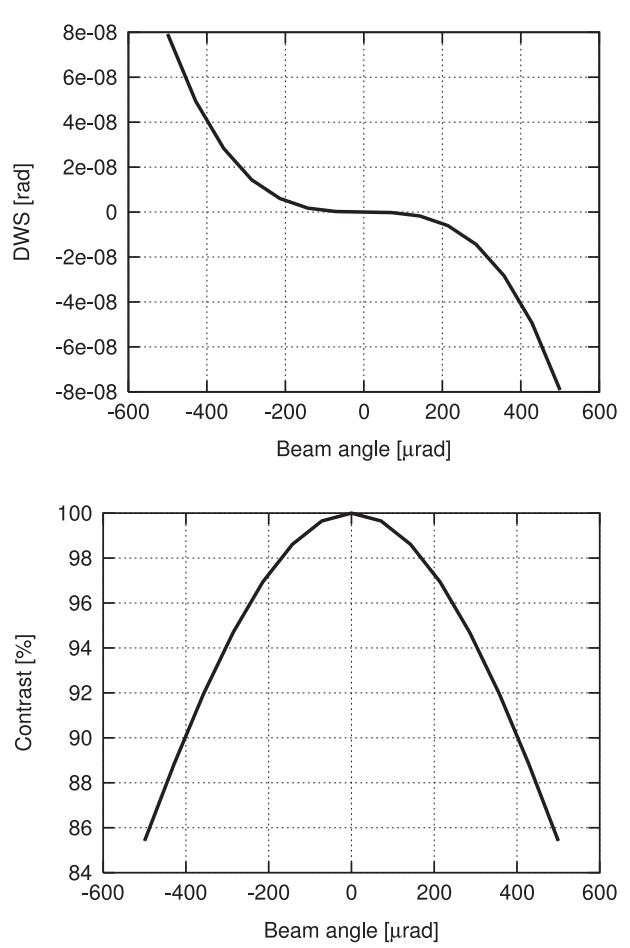

\subsection{A system with zero LPS and DWS response}

The phasefront of a Gaussian beam can locally be approximated by a section of a sphere which is defined by its center and radius of curvature. If the pivot of a tilting beam coincides with the center of this sphere, the beam rotation maps a spherical phasefront upon itself (cf. Fig. 9). In that case, the beam tilt will not cause a phase shift on any quadrant of the QPD. Thus, the phase signal and the DWS signal should not sense the rotation. Such a system is given by setting the distance between pivot (test mass) and QPD as twice the Rayleigh range. The beam waist is centered between test mass and QPD. The resulting signals for the settings listed in Table 5 are shown in Fig. 10. The longitudinal pathlength signal (LPS) and DWS signal show indeed a negligible response in the order of femtometers and nanoradians.

It has therefore been shown for this example, that the numerical procedures described in this paper generate an LPS and DWS response that agrees with intuitive expectations.

\section{Conclusions}

This paper describes the methods to predict the interferometer alignment signals LPS, DWS, DPS and the contrast. The simulations include tracing Gaussian beams through typical optical setups consisting of mirrors, beam splitters and lenses up to a detector. On the detector surface, the photocurrent is generated while taking into account the finite detector size and possibly slits (like in a quadrant photodiode). Once the photocurrent is generated, the same phase demodulation procedure can be used as in a corresponding experiment. Finally, methods to define alignment signals were presented. For examples, a comparison was presented to experimental results, as far as possible analytical results and intuitively clear results. All comparisons showed good agreement.

The methods and functions described in this paper are collected in both C- and C++-libraries called "IfoCad" which act as 
toolbox for investigating and optimizing arbitrary interferometers. IfoCad is available on request from the authors.

\section{Acknowledgments}

We gratefully acknowledge the LTP working group at the University of Glasgow for providing the measurement results listed in Table 2 . We gratefully acknowledge support by Deutsches Zentrum für Luft- und Raumfahrt (DLR) with funding of the Bundesministerium für Wirtschaft und Technologie with a decision of the Deutschen Bundestag (DLR project reference no. 50 OQ 0601) and thank the Deutsche Forschungsgemeinschaft (DFG) for funding the Cluster of Excellence QUEST_Centre for Quantum Engineering and Spacetime Research.

\section{References}

[1] F. Antonucci, M. Armano, H. Audley, et al., Classical and Quantum Gravity 28 (2011) 094001.

[2] O. Jennrich, Classical and Quantum Gravity 26 (2009) 153001. (32 pp.).

[3] J. Hough, Progress in Particle and Nuclear Physics 66 (2011) 233.

[4] M. Dehne, et al., Journal of Physics: Conference Series 154 (2009) 012023.

[5] R. Pierce, J. Leitch, M. Stephens, P. Bender, R. Nerem, Applied Optics 47 (27) (2008).

[6] G. Heinzel, V. Wand, A. Garciá, O. Jennrich, C. Braxmaier, D. Robertson, K. Middleton, D. Hoyland, A. Rüdiger, R. Schilling, U. Johann, K. Danzmann, Classical and Quantum Gravity 21 (2004) 581.
[7] E. Morrison, B.J. Meers, D.I. Robertson, H. Ward, Applied Optics 33 (22) (1994) 5041.

[8] E. Morrison, B.J. Meers, D.I. Robertson, H. Ward, Applied Optics 33 (22) (1994) 5037.

[9] Y. Surrel, Topics in Applied Physics 77 (2000) 55

[10] J.H. Bruning, et al., Applied Optics 13 (11) (1974).

[11] F. Cassaing, Comptes Rendus de l'Académie des Sciences Paris t. 2, Serie IV (2001) 87.

[12] K. Freischlad, C.L. Koliopoulos, Journal of the Optical Society of America A 7 (4) (1990).

[13] Y. Surrel, Applied Optics 32 (19) (1993).

[14] Y. Surrel, Applied Optics 36 (1) (1997).

[15] B.E.A. Saleh, M.C. Teich, Elementary electromagnetic waves, in: Fundamentals of Photonics Wiley Series in Pure and Applied Optics, 1991, pp. 169-174.

[16] B.E.A. Saleh, M.C. Teich, Properties of semiconductor photodetectors, in: Fundamentals of Photonics Wiley Series in Pure and Applied Optics, 1991, pp. 648-654.

[17] A. Yariv, The Gaussian beam in a homogeneous medium, in: Quantum Electronics, third ed., John Wiley \& Sons, 1989, pp. 116-120.

[18] B.E.A. Saleh, M.C. Teich, The Gaussian beam, in: Fundamentals of Photonics Wiley Series in Pure and Applied Optics, 1991, pp. 81-92.

[19] J. Berntsen, T. Espelid, A. Genz, ACM Transactions on Mathematical Software (TOMS) 17 (1991) 452.

[20] G. Hechenblaikner, R. Gerndt, et al., Applied Optics 49 (29) (2010).

[21] R. Schilling, OptoCad 〈http://www.rzg.mpg.de/ros/OptoCad/ 〉.

[22] G.A. Massey, A.E. Siegmann, Applied Optics 8 (5) (1969).

[23] G. Wanner, Complex Optical Systems in Space: Numerical Modelling of the Heterodyne Interferometry of LISA Pathfinder and LISA, Dissertation at the Leibniz University of Hanover.

[24] G. Hechenblaikner, Journal of the Optical Society of America A 27 (9) (2010). 